\title{
Media for the detection and recognition of the enteropathogen Providencia alcalifaciens in faeces
}

\author{
B. W. SENIOR
}

Department of Medical Microbiology, Dundee University Medical School, Ninewells Hospital, Dundee DD1 $95 Y$

\begin{abstract}
A medium (PAM: Providencia alcalifaciens medium) is described that enables the presence of the enteropathogen $P$. alcalifaciens in faeces to be detected with ease and simplicity. This organism is probably the only oxidase-negative organism likely to be present in tetrathionate broth cultures of faeces that is unable to ferment the mannitol, xylose or galactose present in the medium. Thus the red colonies of $P$. alcalifaciens appeared quite distinct from the lemon-yellow acid-forming colonies of all the other bacteria that ferment one or more of these sugars. Extensive tests showed the medium to be both highly specific and sensitive in detecting $P$. alcalifaciens. Two additional media are described that enable the identity of presumptive $P$. alcalifaciens isolates to be confirmed unequivocally and with ease.
\end{abstract}

\section{Introduction}

Over many years, several groups [1-5] have believed the Providence group of bacteria to cause diarrhoea in man, and particularly in young infants. On the other hand, Graber and Lincoln [6] found only one such isolate in 607 stools of infants aged $<1$ year in Denver with diarrhoea. It has been thought by others that Providencia spp. are normal intestinal commensals that flourish only during infections caused by bacterial or viral enteropathogens.

The significance of many of these old studies is difficult to evaluate because examinations were not then made for the presence of bacteria such as Campylobacter jejuni and Yersinia enterocolitica and viruses such as rotavirus, which are now known to cause diarrhoea. Moreover, the identification of an isolate as Providencia sp. as opposed to another member of the Proteeae was not so clearly defined then as it is now.

More recent studies have shown that $P$. alcalifaciens was found seven-to-eight times more frequently in the stools of British adults with diarrhoea who had travelled abroad than in those who had not [7]. Albert et al. [8, 9] have shown that $P$. alcalifaciens strains

Received 27 Aug. 1996; accepted 14 Oct. 1996.

Corresponding author: Dr B. W. Senior. have the ability to invade intestinal mucosa and other cell types, cause diarrhoea in rabbits, and bring about actin condensation in a manner similar to that caused by Shigella flexneri. Guth and Perrella [10] have confirmed that isolates of $P$. alcalifaciens from cases of diarrhoea have the ability to invade cells and cause actin condensation whereas other isolates of $P$. alcalifaciens from non-diarrhoeal stools do not. The genetic determinant(s) for invasiveness appeared to be different from those of invasive Shigella spp. and Escherichia coli and were not plasmid-borne [10]. As a preliminary to studies on the enteropathogenicity of $P$. alcalifaciens, a medium which facilitates the detection of this organism in specimens of faeces is described here.

\section{Materials and methods}

\section{Providencia alcalifaciens medium (PAM)}

The medium contained $10 \mathrm{~g}$ of either Lab Lemco powder (Oxoid L29) or Bacto Tryptone (Difco), sodium deoxycholate $5 \mathrm{~g}$, anhydrous disodium hydrogen orthophosphate $0.8 \mathrm{~g}$, phenol red dye $80 \mathrm{mg}$ and agar $12 \mathrm{~g}$ in $1 \mathrm{~L}$ of distilled water. The medium was sterilised in $500-\mathrm{ml}$ volumes at $121^{\circ} \mathrm{C}$ for $15 \mathrm{~min}$. Before use, xylose $1.5 \mathrm{~g}$, mannitol $1.5 \mathrm{~g}$ and galactose $1.5 \mathrm{~g}$ were added to $500 \mathrm{ml}$ of solidified sterile medium and steamed for c. $30 \mathrm{~min}$ to sterilise the sugars and melt the agar. When molten and cool the complete medium was poured into petri dishes. 


\section{Sugar-supplemented phenylalanine deaminase agar}

This contained Tryptone (Oxoid CM 87) 1.5 g, Lphenylalanine $1 \mathrm{~g}$, agar $1.3 \mathrm{~g}$ and bromocresol purple dye $(0.08 \%) 2.5 \mathrm{ml}$ in $100 \mathrm{ml}$ of distilled water. After sterilisation at $121^{\circ} \mathrm{C}$ for $15 \mathrm{~min}$ and when molten but cool, the medium was aseptically supplemented with trehalose, maltose and inositol, each to a final concentration of $0.5 \%$ from sterile (steamed for $1 \mathrm{~h}$ ) stock sugar solutions $(10 \% \mathrm{w} / \mathrm{v}$ in water $)$ and dispensed either in 200- $\mu 1$ amounts into the wells of a microtitration plate or in 2-ml amounts into screwcapped bottles and left to solidify as a slope.

\section{Adonitol peptone water}

This contained Peptone (Oxoid CM 9) $1.5 \mathrm{~g}$ and bromocresol purple dye $(0.08 \%) 2.5 \mathrm{ml}$ in $100 \mathrm{ml}$ distilled water. After sterilisation at $121^{\circ} \mathrm{C}$ for $15 \mathrm{~min}$ and when cool, the medium was aseptically supplemented with adonitol to $0.5 \%$ from a sterile (steamed for $1 \mathrm{~h})$ stock solution $(10 \% \mathrm{w} / \mathrm{v})$ in water.

\section{Analysis of faeces for P. alcalifaciens}

A specimen of faeces (about the size of a small pea) from patients under investigation for infection with intestinal microbial pathogens was inoculated into $10 \mathrm{ml}$ of Tetrathionate Broth (Oxoid). Separate tetrathionate broths were also inoculated with pure cultures of clinical isolates of $P$. alcalifaciens. After overnight incubation at $37^{\circ} \mathrm{C}$, the tetrathionate broth cultures of faeces were plated out on PAM (and other media for detection of other bacterial enteropathogens). In some instances, and blind to the operator, a known volume of a faecal tetrathionate broth culture was supplemented with 0.1 volume of a tetrathionate broth culture of $P$. alcalifaciens before plating out on PAM.

After incubation of the PAM plates overnight at $37^{\circ} \mathrm{C}$, they were examined for the presence of red colonies. Red colonies were tested for oxidase activity and those that were oxidase-negative were picked off and subcultured in $2 \mathrm{ml}$ of nutrient broth for a few hours at $37^{\circ} \mathrm{C}$. The sugar-supplemented phenylalanine deaminase agar and a tube of adonitol peptone water were then inoculated with the nutrient broth subculture and both were incubated overnight at $37^{\circ} \mathrm{C}$.

If the bromocresol purple dye in the sugar-supplemented phenylalanine deaminase medium remained blue, it indicated that none of the sugars trehalose, maltose or inositol present in the medium had been fermented to acid. Formation of phenylpyruvic acid was then demonstrated by the development of a dark green colour within a few moments of the addition to the slope of a few drops of ferric chloride solution $(10 \% \mathrm{w} / \mathrm{v})$ in water. Only isolates of $P$. alcalifaciens form both phenylpyruvic acid from phenylalanine and acid from adonitol but not from trehalose, maltose or inositol.

\section{Results}

The specificity of PAM for detecting $P$. alcalifaciens was investigated initially by culturing on it a variety of organisms likely to be present in tetrathionate broth cultures of faeces from man. These included isolates of Salmonella spp., Escherichia coli, Citrobacter freundii, Klebsiella pneumoniae, Enterobacter cloacae, Ent. aerogenes, Hafnia alvei, Proteus mirabilis, Pr. vulgaris, Morganella morganii and all species of Providencia. All gave rise to lemon-yellow or white colonies except for those of $P$. alcalifaciens which were red.

An examination by culture on PAM of 80 coded (code unknown to operator) pure cultures showed that, in every instance that a culture gave rise to red colonies on PAM, subsequent secondary tests proved these colonies to be $P$. alcalifaciens. The cultures examined comprised 16 isolates of $P$. rettgeri, 12 isolates of $P$. rustigianii, six isolates of $P$. heimbachae (kindly donated by Professor H.E. Muller), 9 isolates of $M$. morganii, and 14 isolates of $P$. stuartii - all organisms thought to be likely to cause confusion with $P$. alcalifaciens by similarity or ability to acidify only one of the sugars in PAM - and 23 strains of $P$. alcalifaciens acidifying none. All the $23 P$. alcalifaciens strains tested gave rise to red colonies on PAM and all 57 isolates of the other organisms gave rise to lemon-yellow or white colonies.

Of 200 faecal specimens examined from patients either with symptoms of diarrhoea or under investigation for infection with enteropathogenic microorganisms, 12 were found to be infected with Campylobacter spp. and seven with Salmonella spp. Bacterial enteropathogens were not detected in the remainder. Of the 200 tetrathionate broth cultures plated out on PAM and incubated overnight at $37^{\circ} \mathrm{C}$, 14 gave rise to red colonies that were oxidase-positive. API 20NE tests subsequently confirmed all these as being isolates of Pseudomonas aeruginosa.

Four tetrathionate broth cultures gave rise on PAM to oxidase-negative colonies that were peach-coloured rather than red. Nevertheless, these were examined and the secondary tests confirmed that none was $P$. alcalifaciens. Further studies showed that after incubation for a further $24 \mathrm{~h}$ on PAM, the colonies of all four cultures had become more yellow and API $20 \mathrm{E}$ tests showed that all four were isolates of $M$. morganii.

Of the 47 tetrathionate broth cultures seeded blind with $P$. alcalifaciens before culture on PAM, red colonies that were oxidase-negative were detected in 
all 47 of them (i.e., $100 \%$ sensitivity). One representative colony from each specimen on subsequent testing on sugar-supplemented phenylalanine deaminase agar and adonitol peptone water sugar medium gave reactions which, in every instance, showed unequivocally that the isolate was $P$. alcalifaciens. Thus PAM appears to be a sensitive and specific medium for detecting the enteropathogen $P$. alcalifaciens in faeces.

\section{Discussion}

There is now good evidence that some strains of $P$. alcalifaciens cause diarrhoea in man $[8,10]$. Therefore, a method is needed that will enable this organism to be detected reliably with ease, sensitivity and accuracy. The media and methods described appear to fulfil these criteria. Because $P$. alcalifaciens, when found in stools, is not always the predominant organism [10], the starting material was a tetrathionate broth culture of the faeces to maximise the sensitivity of isolation of the organism. $P$. alcalifaciens isolates grow well in this medium [11].

The PAM culture medium distinguishes $P$. alcalifaciens from almost all other bacteria capable of growing in tetrathionate broth because unlike the latter organisms it is unable to form acid from any of the constituent sugars of the medium. Therefore, $P$. alcalifaciens colonies appeared red (alkaline) against a background of lemon-yellow or white (acid-forming) colonies of other bacteria.

PAM contains three sugars: xylose, mannitol and galactose. These sugars were chosen, because they are not fermented by $P$. alcalifaciens and because two or more of them are fermented by virtually all other faecal bacteria capable of growth in tetrathionate broth. Thus possible confusion that could arise by detection of variants unable to acidify a sugar was minimised and was not experienced. The exceptions are non-fermentative organisms, $M$. morganii and all Providencia spp. apart from $P$. rettgeri. These latter organisms ferment only one of the sugars in the medium, galactose, yet they did not give rise to false positive red colonies. Although Proteus spp. do not swarm on Lab-Lemco agar-based media, sodium deoxycholate was nevertheless a necessary ingredient in the medium to make it selective. The medium was buffered with phosphate to an alkaline $\mathrm{pH}(c .7 .8)$ to counteract the high acidity formed by the organisms other than $P$. alcalifaciens.

Despite examination of 200 clinical faecal samples (only 13 were from children under 1 year) for $P$. alcalifaciens by conventional methods, both directly and also after tetrathionate broth enrichment, and also by examination of tetrathionate broth cultures on PAM, this organism was not detected. This shows that
$P$. alcalifaciens is not a common enteropathogen of adults and also confirms the importance of the need for the use of a medium such as PAM to facilitate its detection.

However, because of this, in order to determine the specificity of the medium, it was necessary to seed blind some of the tetrathionate broth cultures with $P$. alcalifaciens. The PAM medium appears to be very sensitive because in every instance when $P$. alcalifaciens was added to broths, even in small amounts (routinely at 1 in 10 , but also on occasions at up to 1 in a 1000 dilution in faecal tetrathionate broth cultures), the organism was detected.

Although several of the samples of faeces tested were found to contain other enteropathogens such as Campylobacter and Salmonella spp., the only occasion that red colonies were found on PAM was when $P$. aeruginosa (oxidase positive) or $P$. alcalifaciens (oxidase negative) was present. Thus the medium is also highly specific and the detection of a red, oxidase-negative colony on PAM is almost certainly indicative of $P$. alcalifaciens. This was readily confirmed by the additional supplementary tests.

The sugar-supplemented phenylalanine deaminase agar together with the adonitol peptone water were devised to permit the most important confirmatory tests for $P$. alcalifaciens to be made on the red, oxidase-negative colonies from PAM in the simplest way. The sugars in these media were chosen not only to be discriminatory for $P$. alcalifaciens but those in the phenylalanine deaminase agar were also selected as sugars that are acidified by most of the organisms listed above, which fermented only galactose in PAM, in order to avoid confusion through the detection of sugar-negative variants. It should be noted that if one or more of the sugars in the sugar-supplemented phenylalanine deaminase agar is fermented, not only is the isolate not $P$. alcalifaciens, but the acid conditions prevent further testing of the medium for phenylalanine deaminase activity [12].

\section{References}

1. Stuart CA, Wheeler KM, McGann V. Further studies on an anaerogenic paracolon organism, type 29911. J Bacteriol 1946; 52: $431-438$.

2. Singer J, Bar-Chay J. Biochemical investigation of Providence strains and their relationship to the Proteus group. $J$ Hyg 1954; 52: $1-8$.

3. Ridge LEL, Thomas MEM. Infection with the Providence type of paracolon bacillus in a residential nursery. $J$ Pathol Bacteriol 1955; 69: 335-337.

4. Prakash O, Dayal S, Kalra SL. Bacterial aetiology of infantile diarrhoea in a village population with observations on some Providence strains isolated from diarrhoea and non-diarrhoea cases. Ind J Med Res 1966; 54: 705-713.

5. Bhat P, Myers RM, Feldman RA. Providence group of organisms in the aetiology of juvenile diarrhoea. Ind $J \mathrm{Med}$ Res 1971; 59: 1010-1018.

6. Graber CD, Lincoln AF. Infantile diarrhea in the Denver area: 
significance of Proteus-Providence organisms. Pediatrics 1955; 16: $585-589$

7. Haynes J, Hawkey PM. Providencia alcalifaciens and travellers' diarrhoea. BMJ 1989; 299: 94-95.

8. Albert MJ, Alam K, Ansaruzzaman M et al. Pathogenesis of Providencia alcalifaciens-induced diarrhea. Infect Immun 1992; 60: $5017-5024$

9. Albert MJ, Ansaruzzaman M, Bhuiyan NA, Neogi PKB, Faruque ASG. Characteristics of invasion of Hep-2 cells by Providencia alcalifaciens. J Med Microbiol 1995; 42:
$186-190$

10. Guth BEC, Perrella E. Prevalence of invasion ability and other virulence-associated characteristics in Providencia alcalifaciens strains isolated in Sao Paulo, Brazil. J Med Microbiol 1996; 45: $459-462$.

11. Kreig NR, Holt JG (eds). Bergey's Manual of systematic bacteriology, vol 1. London, Williams and Wilkins. 1984: 495.

12. Senior BW. Media and tests to simplify the recognition and identification of members of the Proteeae. J Med Microbiol 1997; 46: 39-44. 